\title{
An Electrical Conductimetric Study of Association Phenomena of 4- Aminoantipyrine (4-AAp) Complexes in Mixtures of Methanol-Water at Different Temperatures
}

\author{
Yaser O. Hameed \\ Department of Chemistry \\ College of Science \\ University of Mosul
}

\author{
Hala A. Abdel-Rahman \\ Shakir M. Saied \\ Department of Pharmacy \\ Institute of Technical Mosul \\ Technical Foundation
}

(Received 7/6/2011;Accepted 31/10/2011)

\begin{abstract}
The aim of this work is to measure the electrical conductivity of Co (II) and Ni (II) complexes with 4-amino antipyrine in mixtures of different percentages of methanol and water at different temperatures. The data were analyzed using the Lee-Wheaton equation for unsymmetrical electrolytes, the conductivity parameters: ionic equivalent conductivity $\lambda_{\mathrm{M}}{ }^{+2}$, association constant $\mathrm{K}_{\mathrm{A}}$, and the distance parameter $\mathrm{R}$ are calculated. The ionic equivalent conductivity $\lambda_{\mathrm{M}}{ }^{+2}$, increases with increasing temperature, which may attributed to the breaking of hydrogen bonds between solvated ion. Association constant $\mathrm{K}_{\mathrm{A}}$ increase with increasing temperature, which assumed the presences of simple coloumbic interactions between hard sphere ions in continuous medium. The distance parameter $\mathrm{R}$ are calculated at the bist fit values of standard deviation $\sigma \Lambda$. Thermodynamic parameters from the relation between $\mathrm{LnK}_{\mathrm{A}}$ and $1 \backslash \mathrm{T}$ were calculated to examine the nature of interaction. Walden product were calculated which show the variation depending on electrochemical equilibrium between ions and the solvent molecules with the composition of the mixed polar solvents.
\end{abstract}

Keywords: Electrical conductivity, 4-amino antipyrine, Lee-Wheaton equation, Thermodynamic parameters, Walden product. 


\section{درلسة التوصيلية الكهرائية لالهرة التجمع الايوفي لمقدات 4 -لمينو الت بليرين في مزيج من الميثلنط والماء وسرجلت حرارية مختافة}

\section{الماخص}

يتضمن البهث درلسة التوصيلية الكهربائية لمعقدات النيكل والكوبلت مع 4-أمينو التتببايرين في نسب مختلفة من مزيج الميثانول والماء وفي درجلت حرارية مختلفة م. تحليل النتائج بلستخدلم معادلة لـ مي -

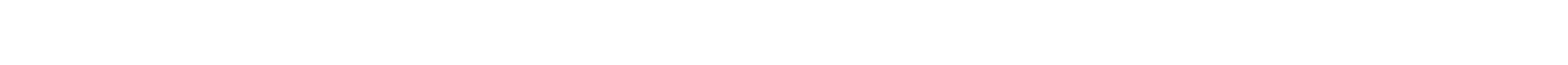

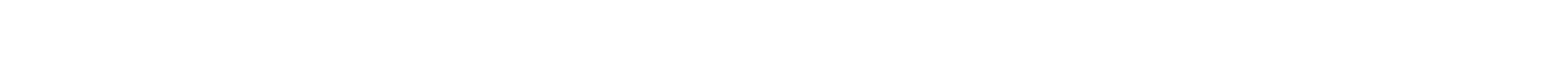

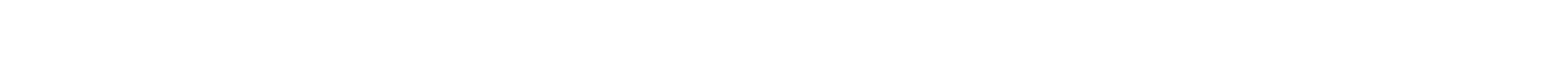

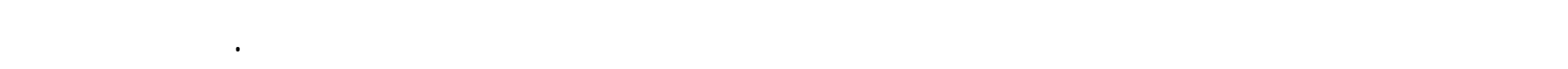

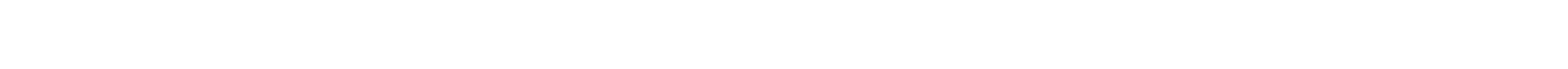

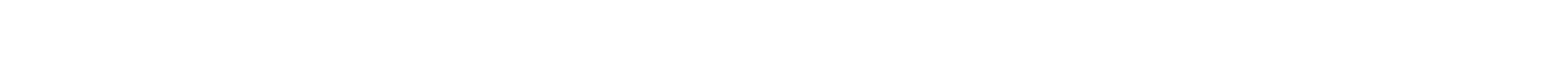
والذي يبين الاختلاف بين التوازن الكهروكيميائي للايونات مع جزيئلت المذيب المتكون من مزيج من منيبين قطبين. الكاملت الدالة: التوصيلية الكهربائية، 4 -أمينو النتيبايرين، معادلة لي -ويتون، الدول الثرمودينلميكية، ن اتج والدن.

\section{INTRODUCTION}

Conductivity measurements are useful as an effective means to understand the nature of solute - solvent interaction, science the degree of ion pairing and ionic mobility is exceedingly sensitive to interaction. The characteristics of metal chelat electrolytes, is of their solute - solvent interaction concerning charge, size and chemical properties of ligand have been elucidated by the study of electronic spectra (Fukuda and Sone, 1972), racemization (Von Meter and Newman, 1976), optical resolution (Iwamoto et al., 1977), viscosity and molal volume (Tominago, 1975) and conductivity(Newmann et al., 1979)( Ito, et al., 1982). The Molar conductivities $(\Lambda)$ of solution of $n$-tetrabutylammomium tetraphenylborate $\left(\mathrm{NBu}_{4} \mathrm{Bph}_{4}\right)$ in 3- pentanone has been measured in the temperature range from 283.15 to $329.15 \mathrm{~K}$., the conductance data have been analyzed using Lee-Wheaton conductivity equation with distance parameter $(\mathrm{R})$ set at Pjerrum's pairing distance, the limiting molar conductivities $\left(\Lambda^{\circ}\right)$ and derived association equilibrium constant $\left(\mathrm{K}_{\mathrm{A}}\right)$ (Tsierkezos, 2007).

In this work we have measured the electrical conductivity of 4-amino antipyrine complexes at different temperatures; Lee-Wheaton equation is used to elucidate the conductivity parameters and $\mathrm{R}$. The calculation of association constants $\left(\mathrm{K}_{\mathrm{A}}\right)$ at different temperature enable calculation of the thermodynamic quantities $(\Delta \mathrm{H}, \Delta \mathrm{Gand} \Delta \mathrm{S})$ for the 
association reaction which can provide detailed information concerning ion-ion and ionsolvent interaction particularly from thermodynamic point of view.

\section{Preparation of complexes}

\section{EXPERIMENTAL}

A mixture of the ligand (4--AAp) (0.378 g, 20 mmole), for 1:2 molar ratio $(0.567 \mathrm{gm}$, 30 mmole) for the 1:3 molar ratio and appropriate metal salt $(10 \mathrm{mmole})$ was refluxed in absolute methanol $(100 \mathrm{ml})$ with continuous stirring for two hours. The solid product formed was filtered of, recrystallized from ethanol, and dried under vacuum. IR spectra were recorded on Unicom SP 2000 spectrometer at the range $\left(700-4000 \mathrm{~cm}^{-1}\right)$ using Nujol mall and $\mathrm{KBr}$ discs, electronic spectra were recorded on a shimadzu UV/Vis spectrophotometer UV-160 for $10^{-3} \mathrm{M}$ solution of the complexes in ethanol at ambient temperature $(25 \mathrm{C})$. Heat of formation and steric energies of products in addition to the geometry (three dimensional structure) of complexes (11) at minimized energy (mm2) were established by chem..3D ultra; molecular modeling and analysis (version 6.0.3) by Pentium (IV) computer (Jonan et al., 2011).

\section{Purification of solvent}

Methanol was purified and dried by the method of Perrin (Perrin et al., 1966) and the procedure was repeated twice to ensure that all water was removed. Conductivity water was prepared by redistilling distilled water three times and condensing it by using a condenser with glass joints, little potassium permanganate was added to the distilled water to reduce the absorption of carbon dioxide (Daniels et al., 1970).

\section{General procedure}

General method has been used for measuring the conductance of the electrolytes. The conductivity cell was weighted and kept at a constant temperature then purified nitrogen passed through the cell: $100 \mathrm{ml}$ of purified solvent was added and nitrogen gas was passed for further (10-15) minutes, where upon the cell plus its content were weighed. Certain amount of complex solution was injected in to the cell from a- plastic syringe (which was weighed before and after each addition), nitrogen gas was passed for several minute and the conductivity of the solution was measured. After performing all additions (generally 15), the cell was reweighed ed to find the weight change over the whole run which was found not to exceed than $(0.02 \%)$.

\section{RESULTS AND DISCUSSION}

The equation derived by Lee-Wheaton has been tested extensively in both aqueous and non-aqueous systems and provide satisfactory explanation of the conductmetric behavior of variety of systems. Lee and Wheaton derived an equation for unsymmetrical electrolytes of the form: 
$\lambda_{\mathrm{j}}=\lambda_{\mathrm{j}}\left\{1+\mathrm{Z}_{\mathrm{j}} \sum_{\mathrm{p}=\mathrm{s}}^{\mathrm{s}} \mathrm{X}_{\mathrm{j}}^{\mathrm{p}} \sum_{\mathrm{v}=1}^{\mathrm{s}} \mathrm{tv} \mathrm{X}_{\mathrm{v}}^{\mathrm{p}}\left[\mathrm{A}_{\mathrm{v}}^{\mathrm{p}}(\mathrm{t})(\beta \mathrm{k})+\mathrm{B}_{\mathrm{v}}^{\mathrm{p}}(\mathrm{t})(\beta \mathrm{k})^{2}+\mathrm{C}_{\mathrm{v}}^{\mathrm{p}}(\mathrm{t})(\beta \mathrm{k})^{3}\right]\right\}-$

$\mathrm{Z}_{\mathrm{j}}(\mathrm{kt}) / 2(1+\mathrm{t})\left\{1+\mathrm{v}_{\mathrm{j}}^{1}(\mathrm{t})(\beta \mathrm{k})+\mathrm{v}_{\mathrm{j}}^{2}(\mathrm{t})(\beta \mathrm{k})^{2}+\pi_{\mathrm{j}}^{5} \frac{\mathrm{t}}{6}\right\}-------(1)$

With $\Lambda_{\text {equiv. }}=\sum_{j=1}^{s}\left(\left|z_{j}\right| m_{j} \lambda_{j} / C\right)$ where $s$ is the number of charged species, $z_{j}$ and $t_{j}$ are the charge and transference number of species

$\beta=\mathrm{e}^{2} / \mathrm{DKT}, \mathrm{k}=\left(4 \pi / \mathrm{DKT} \sum_{\mathrm{j}=1}^{\mathrm{s}} \mathrm{n}_{\mathrm{j}} \mathrm{e}_{\mathrm{j}}{ }^{2}\right.$ and is proportional to the ionic strength. $\mathrm{t}=\mathrm{KR}$ and $\mathrm{T}=\mathrm{Fe} / 6 \pi \eta, \mathrm{n}_{\mathrm{j}}$ is the molar free ion concentration of species $\mathrm{j}$. $\mathrm{C}$ is the equivalent stoichiometric concentration of the electrolyte. The plasma coefficients $A_{v}^{p}, B_{v}^{p},------$ etc are functions of KR and $\mathrm{q}_{\mathrm{p}}$ while the terms $\mathrm{x}_{\mathrm{j}}^{\mathrm{p}}$ and $\mathrm{q}_{\mathrm{p}}$ are functions of the limiting mobility's, the concentration and charge of all ions present in solution [all other terms are defined in the original paper] (Lee and Wheaton, 1978).

For an unsymmetrical electrolyte $\mathrm{MX}_{2}$ ionizing in to $\mathrm{M}^{+2}$ and $\mathrm{X}^{-}$the possible association equilibrium are

$\mathrm{M}^{2+}+\mathrm{X}^{-}--\mathrm{kA}^{(1)}---\mathrm{MX}^{+}$

and

$\mathrm{MX}^{+}+\mathrm{X}^{-}--\mathrm{kA}^{(2)}---\mathrm{MX}_{2}---(3)$

Thus three ionic species are present in the solutions which are $\mathrm{MX}^{+}, \mathrm{M}^{2+}$, and $\mathrm{X}^{\sim}$.

Thus for associated salts:

$\Lambda_{\mathrm{MX} 2}=f\left(\lambda_{\mathrm{M}^{2+}}^{\mathrm{o}}, \lambda_{\mathrm{MX}^{+}}^{\mathrm{o}}, \lambda_{\mathrm{X}^{-}}^{\mathrm{o}}, \mathrm{K}_{\mathrm{A}}^{(1)}, \mathrm{K}_{\mathrm{A}}^{(2)}, \mathrm{R}\right)$

Where $\mathrm{R}$ is the average center-to-center distance for ion pairs.

The input data to the computer program $\left(\mathrm{RM}_{11}\right)$ are solvent data (temp. T., dielectric constant $\mathrm{D}$ and viscosity $\eta$ ); the charge $\mathrm{Zi}$ and ionic mobility $\lambda_{\mathrm{i}}$ for each ionic species, $\mathrm{K}_{\mathrm{A}}^{(1)}, \mathrm{K}_{\mathrm{A}}^{(2)}, \lambda_{\mathrm{mx}^{+}}, \lambda_{\mathrm{m}^{2+}}^{\cdot}$ and $\mathrm{R}$ all in the form $K_{A}^{(1)}(\min ), K_{A}^{(2)}(\max ), \Delta K_{A}-$ etc, then the experimental data (molecular concentration and the equivalent conductance). This program is used to determine values of $\left(\mathrm{K}_{\mathrm{A}}^{(1)}, \mathrm{K}_{\mathrm{A}}^{(2)}, \lambda_{\mathrm{mx}^{+}}, \lambda_{\mathrm{m}^{2+}}\right.$ and $\mathrm{R}$ which minimize $\sigma_{\mathrm{s}}(\Lambda)$.

Table (1) (A-H) shows the molar concentration and equivalent conductance's of [Ni (4AAp) $)_{3} \mathrm{Cl}_{2}$ in different percentages of methanol water at different temperatures (293.15313.15 K), and Fig. $1(\mathrm{~A}-\mathrm{H})$ illustrates the relation between them. From both tables and figures it can be seen clearly that the equivalent conductivities increase with increasing temperatures and increasing the percentages of methanol, this is because of the effect of viscosity which play an important role which may be due to the contribution of triple ions which are expected to be present in the solution together with the free ions (Hikmat, 2002), generally the equivalent conductance of $\mathrm{Co}^{2+}$ complexes more than that of $\mathrm{Ni}^{2+}$ complexes and this is related to the ionic radii of $\mathrm{Co}$ which is more than $\mathrm{Ni}$ and form small solvated ion in solution. 
Table $1 \mathrm{~A}$ : Molar concentration (M) and equivalent conductance of [Ni-4-AAp] $\mathrm{Cl}_{2}$ in $50 \%$ methanol-water mixture.

\begin{tabular}{|c|c|c|c|c|c|}
\hline $\begin{array}{c}\text { Conc. } \\
\text { *10-5 M }\end{array}$ & $\mathbf{T = 2 9 3 ~ K}$ & $\mathbf{T = 2 9 8} \mathbf{K}$ & $\mathbf{T}=\mathbf{3 0 3} \mathbf{~ K}$ & $\mathbf{T = 3 0 8} \mathbf{K}$ & $\mathbf{T}=\mathbf{3 1 3} \mathbf{~ K}$ \\
\hline 4.3695 & 80.0877 & 80.4293 & 82.2225 & 89.6471 & 104.3928 \\
\hline 8.3457 & 78.2619 & 79.1752 & 81.2828 & 85.8090 & 94.8153 \\
\hline 12.7402 & 77.4813 & 79.1384 & 81.1347 & 84.4257 & 87.1753 \\
\hline 16.5494 & 77.3524 & 78.9013 & 80.6119 & 83.8332 & 85.2549 \\
\hline 20.7744 & 77.1599 & 78.6678 & 80.2712 & 82.3550 & 84.0159 \\
\hline 24.9281 & 76.2972 & 78.1048 & 79.8603 & 82.1022 & 83.4113 \\
\hline 29.1623 & 75.7246 & 77.5682 & 79.7644 & 82.0781 & 83.1349 \\
\hline 32.9363 & 75.7245 & 77.4370 & 79.4635 & 80.8634 & 82.8410 \\
\hline 37.0007 & 75.4950 & 77.2471 & 78.9221 & 80.7946 & 82.6189 \\
\hline 40.4563 & 73.4408 & 75.6856 & 78.3364 & 80.7240 & 82.4845 \\
\hline 44.4878 & 70.0552 & 73.8384 & 77.9303 & 80.6360 & 82.1206 \\
\hline 48.2247 & 67.7582 & 72.8305 & 76.2996 & 80.3792 & 81.8076 \\
\hline 52.2860 & 57.4113 & 67.9822 & 76.2039 & 80.0644 & 81.5465 \\
\hline 55.8697 & 47.4333 & 49.8273 & 54.6153 & 59.4034 & 63.7924 \\
\hline 59.8184 & 42.6079 & 45.6565 & 48.0954 & 50.5344 & 60.2900 \\
\hline
\end{tabular}

Table $1 \mathrm{~B}$ : Molar concentration (M) and equivalent conductance of [Ni-4-AAp] $\mathrm{Cl}_{2} \mathrm{in} 70 \%$ methanol-water mixture.

\begin{tabular}{|c|c|c|c|c|c|}
\hline $\begin{array}{c}\text { Conc. } \\
\text { *10-5 M }\end{array}$ & $\mathbf{T}=\mathbf{2 9 3} \mathbf{~ K}$ & $\mathbf{T}=\mathbf{2 9 8} \mathbf{~ K}$ & $\mathbf{T}=\mathbf{3 0 3} \mathbf{~ K}$ & $\mathbf{T}=\mathbf{3 0 8} \mathbf{~ K}$ & $\mathbf{T}=\mathbf{3 1 3} \mathbf{~ K}$ \\
\hline 4.3695 & 149.2791 & 151.9671 & 159.5425 & 164.9186 & 170.7834 \\
\hline 8.3457 & 139.3554 & 145.8857 & 152.8241 & 157.3136 & 160.9869 \\
\hline 12.7402 & 130.0708 & 136.3152 & 138.9170 & 143.7738 & 146.6761 \\
\hline 16.5494 & 125.7580 & 128.2712 & 131.1270 & 134.9448 & 145.6818 \\
\hline 20.7744 & 123.6592 & 127.7604 & 130.6444 & 134.7211 & 138.5930 \\
\hline 24.9281 & 123.2462 & 126.1174 & 129.4974 & 133.8687 & 138.0067 \\
\hline 29.1623 & 121.6820 & 125.0594 & 127.0405 & 133.7792 & 137.9475 \\
\hline 32.9363 & 118.8324 & 122.2594 & 126.7482 & 129.2813 & 136.0363 \\
\hline 37.0007 & 118.6159 & 121.1503 & 124.8639 & 128.8296 & 135.9249 \\
\hline 40.4563 & 117.7180 & 120.3666 & 123.6846 & 128.7022 & 131.6033 \\
\hline 44.4878 & 114.9278 & 118.8322 & 122.5587 & 126.0240 & 130.2101 \\
\hline 48.2247 & 108.7998 & 116.9772 & 121.6476 & 124.8420 & 128.4609 \\
\hline 52.2860 & 104.4905 & 112.4706 & 118.7705 & 121.1614 & 126.5773 \\
\hline 55.8697 & 67.6068 & 80.4111 & 96.2641 & 114.9636 & 122.9547 \\
\hline 59.8184 & 50.3641 & 69.1567 & 92.6474 & 107.8489 & 117.6046 \\
\hline
\end{tabular}


Table $1 \mathrm{C}$ : Molar concentration (M) and equivalent conductance of [Ni-4-AAp] $\mathrm{Cl}_{2}$ in $90 \%$ methanol -water mixture.

\begin{tabular}{|c|c|c|c|c|c|}
\hline $\begin{array}{c}\text { Conc. } \\
\text { *10-5 M }\end{array}$ & $\mathbf{T}=\mathbf{2 9 3} \mathbf{K}$ & $\mathbf{T}=\mathbf{2 9 8} \mathbf{~ K}$ & $\mathbf{T}=\mathbf{3 0 3} \mathbf{~ K}$ & $\mathbf{T}=\mathbf{3 0 8} \mathbf{~ K}$ & $\mathbf{T}=\mathbf{3 1 3} \mathbf{~ K}$ \\
\hline 4.3695 & 122.1933 & 124.0200 & 125.5344 & 146.6761 & 178.3886 \\
\hline 8.3457 & 120.8199 & 122.6192 & 125.2987 & 132.2381 & 148.0910 \\
\hline 12.7402 & 120.5654 & 122.6124 & 123.8940 & 127.0340 & 130.7072 \\
\hline 16.5494 & 120.0362 & 122.3569 & 123.8796 & 125.4787 & 128.9520 \\
\hline 20.7744 & 119.5894 & 122.0095 & 123.4970 & 125.4310 & 128.5397 \\
\hline 24.9281 & 118.5403 & 121.0744 & 123.4734 & 125.3779 & 127.8858 \\
\hline 29.1623 & 118.5156 & 120.6265 & 122.6217 & 125.0726 & 126.9681 \\
\hline 32.9363 & 114.9019 & 118.0001 & 121.9986 & 125.0493 & 126.7861 \\
\hline 37.0007 & 114.8434 & 116.5919 & 121.2629 & 124.5980 & 126.0240 \\
\hline 40.4563 & 111.6465 & 116.5020 & 120.9325 & 123.5818 & 125.4123 \\
\hline 44.4878 & 109.9500 & 112.7253 & 120.4774 & 123.0819 & 125.0594 \\
\hline 48.2247 & 101.8075 & 106.0637 & 118.7429 & 120.5865 & 123.6592 \\
\hline 52.2860 & 90.1668 & 105.4100 & 116.0209 & 117.7555 & 119.8369 \\
\hline 55.8697 & 86.5355 & 99.6947 & 109.4078 & 112.1440 & 117.0081 \\
\hline 59.8184 & 80.9021 & 93.7175 & 100.1015 & 104.8895 & 115.2636 \\
\hline
\end{tabular}

Table 1D: Molar concentration (M) and equivalent conductance of [Ni-4-AAp] $\mathrm{Cl}_{2}$ in $100 \%$ methanol.

\begin{tabular}{|c|c|c|c|c|c|}
\hline $\begin{array}{c}\text { Conc. } \\
\text { *10-5 M }\end{array}$ & $\mathbf{T = 2 9 3 ~ K}$ & $\mathbf{T = 2 9 8} \mathbf{K}$ & $\mathbf{T}=\mathbf{3 0 3} \mathbf{~ K}$ & $\mathbf{T}=\mathbf{3 0 8} \mathbf{K}$ & $\mathbf{T}=\mathbf{3 1 3} \mathbf{~ K}$ \\
\hline 4.3695 & 161.4975 & 166.3848 & 173.4715 & 187.0838 & 200.7048 \\
\hline 8.3457 & 151.9717 & 161.6979 & 171.9218 & 179.0266 & 196.2609 \\
\hline 12.7402 & 151.9695 & 161.5477 & 168.0822 & 178.8475 & 185.1068 \\
\hline 16.5494 & 151.1915 & 156.0892 & 163.1196 & 178.3886 & 183.9793 \\
\hline 20.7744 & 143.7738 & 148.2836 & 161.3950 & 176.1386 & 183.4553 \\
\hline 24.9281 & 140.0984 & 146.2619 & 154.1883 & 169.1497 & 172.4148 \\
\hline 29.1623 & 138.2978 & 143.9393 & 153.4873 & 157.1299 & 161.4662 \\
\hline 32.9363 & 133.4030 & 142.4102 & 147.9339 & 153.1575 & 156.9984 \\
\hline 37.0007 & 130.5559 & 140.8034 & 144.3294 & 147.8935 & 149.2643 \\
\hline 40.4563 & 129.1894 & 134.7824 & 136.9140 & 141.0520 & 144.3122 \\
\hline 44.4878 & 122.5263 & 133.0690 & 132.8406 & 136.0391 & 139.6946 \\
\hline 48.2247 & 119.3682 & 125.2705 & 128.4369 & 131.4978 & 133.1865 \\
\hline 52.2860 & 119.1793 & 121.2477 & 123.8796 & 125.7316 & 130.0205 \\
\hline 55.8697 & 118.9083 & 120.8199 & 122.7839 & 125.0895 & 126.9681 \\
\hline 59.8184 & 117.3127 & 120.6406 & 122.5587 & 123.6547 & 126.4860 \\
\hline
\end{tabular}


Table $1 \mathrm{E}$ : The relation between equivalent conductance and square root of concentration of [Co-4-AAp $] \mathrm{Cl}_{2}$ in $50 \%$ methanol -water mixture.

\begin{tabular}{|c|c|c|c|c|c|}
\hline $\begin{array}{c}\text { Conc. } \\
\text { *10-5 M }\end{array}$ & $\mathbf{T}=\mathbf{2 9 3} \mathbf{~ K}$ & $\mathbf{T}=\mathbf{2 9 8} \mathbf{~ K}$ & $\mathbf{T}=\mathbf{3 0 3} \mathbf{~ K}$ & $\mathbf{T}=\mathbf{3 0 8} \mathbf{~ K}$ & $\mathbf{T}=\mathbf{3 1 3} \mathbf{~ K}$ \\
\hline 4.3695 & 80.0877 & 80.4293 & 82.2225 & 89.6471 & 104.3928 \\
\hline 8.3457 & 78.2619 & 79.1752 & 81.2828 & 85.8090 & 94.8153 \\
\hline 12.7402 & 77.4813 & 79.1384 & 81.1347 & 84.4257 & 87.1753 \\
\hline 16.5494 & 77.3524 & 78.9013 & 80.6119 & 83.8332 & 85.2549 \\
\hline 20.7744 & 77.1599 & 78.6678 & 80.2712 & 82.3550 & 84.0159 \\
\hline 24.9281 & 76.2972 & 78.1048 & 79.8603 & 82.1022 & 83.4113 \\
\hline 29.1623 & 75.7246 & 77.5682 & 79.7644 & 82.0781 & 83.1349 \\
\hline 32.9363 & 75.7245 & 77.4370 & 79.4635 & 80.8634 & 82.8410 \\
\hline 37.0007 & 75.4950 & 77.2471 & 78.9221 & 80.7946 & 82.6189 \\
\hline 40.4563 & 73.4408 & 75.6856 & 78.3364 & 80.7240 & 82.4845 \\
\hline 44.4878 & 70.0552 & 73.8384 & 77.9303 & 80.6360 & 82.1206 \\
\hline 48.2247 & 67.7582 & 72.8305 & 76.2996 & 80.3792 & 81.8076 \\
\hline 52.2860 & 57.4113 & 67.9822 & 76.2039 & 80.0644 & 81.5465 \\
\hline 55.8697 & 47.4333 & 49.8273 & 54.6153 & 59.4034 & 63.7924 \\
\hline 59.8184 & 42.6079 & 45.6565 & 48.0954 & 50.5344 & 60.2900 \\
\hline
\end{tabular}

Table 1F: Molar concentration (M) and equivalent conductance of [Co-4-AAp]Cl ${ }_{2}$ in $70 \%$ methanol -water mixture.

\begin{tabular}{|c|c|c|c|c|c|}
\hline $\begin{array}{c}\text { Conc. } \\
\text { *10-5 M }\end{array}$ & $\mathbf{T}=\mathbf{2 9 3} \mathbf{~ K}$ & $\mathbf{T}=\mathbf{2 9 8} \mathbf{~ K}$ & $\mathbf{T}=\mathbf{3 0 3} \mathbf{~ K}$ & $\mathbf{T}=\mathbf{3 0 8} \mathbf{~ K}$ & $\mathbf{T}=\mathbf{3 1 3} \mathbf{~ K}$ \\
\hline 4.3695 & 240.1624 & 257.3267 & 287.0781 & 324.8395 & 351.1580 \\
\hline 8.3457 & 231.1851 & 240.1717 & 259.3433 & 277.9157 & 289.2988 \\
\hline 12.7402 & 216.5905 & 231.8964 & 244.4551 & 253.0892 & 259.7610 \\
\hline 16.5494 & 208.7321 & 214.1703 & 220.8171 & 225.3490 & 231.6936 \\
\hline 20.7744 & 200.6991 & 205.9941 & 212.0111 & 219.2315 & 225.0079 \\
\hline 24.9281 & 185.6434 & 192.9302 & 199.7616 & 202.0387 & 204.9231 \\
\hline 29.1623 & 185.3215 & 193.3799 & 198.0091 & 202.8099 & 206.2389 \\
\hline 32.9363 & 180.8964 & 190.3235 & 194.9368 & 200.7535 & 206.7708 \\
\hline 37.0007 & 179.8454 & 182.6832 & 186.7372 & 189.4398 & 192.2776 \\
\hline 40.4563 & 175.8539 & 178.2021 & 181.6627 & 183.0221 & 185.4940 \\
\hline 44.4878 & 168.2348 & 171.1570 & 174.8659 & 176.7765 & 178.6872 \\
\hline 48.2247 & 162.4563 & 164.8410 & 167.1220 & 169.4030 & 171.0618 \\
\hline 52.2860 & 153.1843 & 157.1050 & 158.9220 & 161.3127 & 163.0340 \\
\hline 55.8697 & 146.2224 & 149.7127 & 152.5765 & 154.0084 & 155.9773 \\
\hline 59.8184 & 139.9970 & 142.1703 & 145.1794 & 147.3526 & 150.0274 \\
\hline
\end{tabular}


Table $1 \mathrm{G}$ : Molar concentration (M) and equivalent conductance of [Co-4-AAp] $\mathrm{Cl}_{2}$ in $90 \%$ methanol-water mixture.

\begin{tabular}{|c|c|c|c|c|c|}
\hline $\begin{array}{c}\text { Conc. } \\
\text { *10-5 M }\end{array}$ & $\mathbf{T = 2 9 3 ~ K}$ & $\mathbf{T}=\mathbf{2 9 8} \mathbf{~ K}$ & $\mathbf{T}=\mathbf{3 0 3} \mathbf{~ K}$ & $\mathbf{T}=\mathbf{3 0 8} \mathbf{~ K}$ & $\mathbf{T}=313 \mathbf{~ K}$ \\
\hline 4.3695 & 212.6996 & 226.4310 & 240.1624 & 259.6153 & 274.4910 \\
\hline 8.3457 & 164.6837 & 174.2695 & 188.6481 & 195.2384 & 204.8242 \\
\hline 12.7402 & 145.3424 & 151.0498 & 158.1140 & 171.4576 & 208.3488 \\
\hline 16.5494 & 144.9931 & 157.6730 & 169.4558 & 183.6557 & 196.0428 \\
\hline 20.7744 & 144.1725 & 157.1358 & 168.9292 & 182.1666 & 192.7566 \\
\hline 24.9281 & 142.5667 & 152.8156 & 162.8445 & 170.6670 & 181.2976 \\
\hline 29.1623 & 141.6307 & 151.8879 & 157.8888 & 163.3754 & 174.3484 \\
\hline 32.9363 & 140.7082 & 146.1732 & 151.0311 & 159.8360 & 171.2216 \\
\hline 37.0007 & 136.8732 & 138.9002 & 142.0083 & 150.7919 & 163.8997 \\
\hline 40.4563 & 133.3938 & 136.1815 & 140.8779 & 152.8661 & 163.2477 \\
\hline 44.4878 & 132.3502 & 134.9673 & 137.4399 & 147.4426 & 156.8834 \\
\hline 48.2247 & 130.9371 & 134.0476 & 134.9807 & 141.7200 & 150.0145 \\
\hline 52.2860 & 127.3648 & 130.9030 & 133.0068 & 136.0669 & 141.9958 \\
\hline 55.8697 & 123.8489 & 126.6233 & 130.9190 & 133.0668 & 136.6466 \\
\hline 59.8184 & 121.2737 & 123.6977 & 127.8770 & 130.4682 & 133.1430 \\
\hline
\end{tabular}

Table $1 \mathrm{H}$ : Molar concentration (M) and equivalent conductance of [Co-4-AAp] $\mathrm{Cl}_{2}$ in $100 \%$ methanol.

\begin{tabular}{|c|c|c|c|c|c|}
\hline $\begin{array}{c}\text { Conc. } \\
\text { *10-5 M }\end{array}$ & $\mathbf{T}=\mathbf{2 9 3} \mathbf{~ K}$ & $\mathbf{T}=\mathbf{2 9 8} \mathbf{~ K}$ & $\mathbf{T}=\mathbf{3 0 3} \mathbf{~ K}$ & $\mathbf{T}=\mathbf{3 0 8} \mathbf{K}$ & $\mathbf{T}=\mathbf{3 1 3} \mathbf{~ K}$ \\
\hline 4.3695 & 232.1525 & 248.1724 & 261.9038 & 292.7995 & 339.7152 \\
\hline 8.3457 & 182.6570 & 192.2428 & 204.2251 & 225.1940 & 244.9646 \\
\hline 12.7402 & 153.0120 & 166.2817 & 178.9144 & 189.5108 & 202.0695 \\
\hline 16.5494 & 145.8901 & 162.8235 & 172.0580 & 182.7493 & 190.9067 \\
\hline 20.7744 & 152.0815 & 159.0335 & 169.1537 & 176.6310 & 187.9430 \\
\hline 24.9281 & 148.8041 & 158.6296 & 168.6612 & 173.6626 & 185.5097 \\
\hline 29.1623 & 146.2299 & 157.3708 & 163.8897 & 171.8289 & 184.6357 \\
\hline 32.9363 & 141.9226 & 151.5067 & 162.8187 & 170.8675 & 173.7644 \\
\hline 37.0007 & 152.5486 & 151.2021 & 156.0795 & 166.8727 & 173.4988 \\
\hline 40.4563 & 145.9451 & 147.3877 & 151.7901 & 162.6298 & 167.3262 \\
\hline 44.4878 & 138.7886 & 142.3851 & 144.2957 & 148.6789 & 153.7365 \\
\hline 48.2247 & 133.3218 & 135.0844 & 138.4022 & 140.5795 & 143.7936 \\
\hline 52.2860 & 127.8429 & 129.7555 & 131.6680 & 134.5369 & 137.0232 \\
\hline 55.8697 & 124.2964 & 125.5493 & 127.1602 & 128.4131 & 130.6505 \\
\hline 59.8184 & 120.0199 & 122.1096 & 123.9485 & 125.9545 & 127.8770 \\
\hline
\end{tabular}


Table 2(A-B) shows the best fit parameters of analysis of conductance data for both complexes in different percentages of methanol and water at different temperatures.

From Table 2(A-B); the values of $\lambda_{\mathrm{M}}{ }^{+2}$ increase with increasing temperatures which may be attributed to the breaking of hydrogen bonds between solvated ion, and because of the large size of the complex ion which form small size of solvated ion to move in solution. The value of association constant $\mathrm{K}_{\mathrm{A}}$ increase with increasing temperature, which assumed simple columbic interactions between hard sphere ions in continuous medium, the same behavior was obtained by Dawood (Dawood, 1996) for symmetrical (1:1) electrolytes in methanol at different temperatures, the values of $\mathrm{R}$ are generally $10 \mathrm{~A}^{\circ}$ which indicate that the ions are separated by solvent molecules (SSIP).

Table $2 \mathrm{~A}$ : Results of analysis of the conductance data for $\left[\mathrm{Ni}(4-\mathrm{AAP})_{3}\right] \mathrm{Cl}_{2}$ in $\mathrm{Methanol} \mathrm{-}$ Water mixture at different temperatures.

\begin{tabular}{|c|c|c|c|c|c|}
\hline $\mathbf{T}\left(\mathbf{K}^{\mathbf{0}}\right)$ & $\mathbf{K}_{\mathbf{A}}$ & $\lambda^{0}{ }_{M}+2$ & $\lambda^{\mathbf{0}} \mathrm{MX}^{+}$ & $\mathbf{R}_{\mathbf{A}} \mathbf{0}$ & $\sigma \Lambda$ \\
\hline \multicolumn{6}{|c|}{$100 \%$ Methanol } \\
\hline 293.16 & 699 & 100 & 0.1 & 10 & 0.081 \\
\hline 298.16 & 650 & 120 & 0.1 & 10 & 0.068 \\
\hline 303.16 & 560 & 130 & 0.2 & 10 & 0.059 \\
\hline 308.16 & 473 & 145 & 0.1 & 10 & 0.056 \\
\hline 313.16 & 400 & 150 & 0.2 & 10 & 0.095 \\
\hline \multicolumn{6}{|c|}{$90 \%$ Methanol } \\
\hline 293.16 & 749 & 50 & 0.1 & 10 & 0.091 \\
\hline 298.16 & 850 & 80 & 0.3 & 10 & 0.064 \\
\hline 303.16 & 943 & 100 & 0.3 & 10 & 0.069 \\
\hline 308.16 & 1100 & 110 & 0.3 & 10 & 0.058 \\
\hline 313.16 & 1200 & 120 & 0.1 & 10 & 0.067 \\
\hline \multicolumn{6}{|c|}{$70 \%$ Methanol } \\
\hline 293.16 & 915 & 110 & 0.1 & 10 & 0.074 \\
\hline 298.16 & 1043 & 120 & 0.1 & 10 & 0.078 \\
\hline 303.16 & 1150 & 125 & 0.1 & 10 & 0.071 \\
\hline 308.16 & 1274 & 130 & 0.1 & 10 & 0.062 \\
\hline 313.16 & 1325 & 140 & 0.1 & 10 & 0.055 \\
\hline \multicolumn{6}{|c|}{$50 \%$ Methanol } \\
\hline 293.16 & 1100 & 55 & 0.1 & 10 & 0.074 \\
\hline 298.16 & 1274 & 50 & 0.2 & 10 & 0.078 \\
\hline 303.16 & 1400 & 45 & 0.2 & 10 & 0.071 \\
\hline 308.16 & 1500 & 35 & 0.1 & 10 & 0.062 \\
\hline 313.16 & 1600 & 30 & 0.1 & 10 & 0.055 \\
\hline
\end{tabular}


Table $2 \mathrm{~B}$ : The results of analysis of the conductance data for $\left[\mathrm{Co}(4-\mathrm{AAP})_{3}\right] \mathrm{Cl}_{2}$ in $\mathrm{Methanol}$ - Water mixture at different temperatures.

\begin{tabular}{|c|c|c|c|c|c|}
\hline $\mathbf{T} /\left(\mathbf{K}^{\mathbf{0}}\right)$ & $\mathbf{K}_{\mathbf{A}}$ & $\lambda^{0}{ }_{M}^{+2}$ & $\lambda^{\mathbf{0}} \mathrm{MX}^{+}$ & $\mathbf{R}_{\mathbf{A}} \mathbf{0}$ & $\boldsymbol{\sigma} \Lambda$ \\
\hline \multicolumn{6}{|c|}{$100 \%$ Methanol } \\
\hline 293.16 & 650 & 150 & 0.1 & 10 & 0.091 \\
\hline 298.16 & 727 & 155 & 0.1 & 10 & 0.082 \\
\hline 303.16 & 790 & 155 & 0.2 & 10 & 0.071 \\
\hline 308.16 & 850 & 160 & 0.2 & 10 & 0.09 \\
\hline 313.16 & 900 & 165 & 0.1 & 10 & 0.072 \\
\hline \multicolumn{6}{|c|}{$90 \%$ Methanol } \\
\hline 293.16 & 1152 & 150 & 0.3 & 10 & 0.087 \\
\hline 298.16 & 1199 & 160 & 0.3 & 10 & 0.061 \\
\hline 303.16 & 1248 & 165 & 0.2 & 10 & 0.083 \\
\hline 308.16 & 1300 & 170 & 0.2 & 10 & 0.092 \\
\hline 313.16 & 1350 & 175 & 0.2 & 10 & 0.072 \\
\hline \multicolumn{6}{|c|}{$70 \%$ Methanol } \\
\hline 293.16 & 900 & 155 & 0.1 & 10 & 0.1 \\
\hline 298.16 & 1000 & 180 & 0.1 & 10 & 0.085 \\
\hline 303.16 & 1100 & 190 & 0.2 & 10 & 0.091 \\
\hline 308.16 & 1150 & 200 & 0.3 & 10 & 0.073 \\
\hline 313.16 & 1200 & 205 & 0.1 & 10 & 0.069 \\
\hline \multicolumn{6}{|c|}{$50 \%$ Methanol } \\
\hline 293.16 & 699 & 150 & 0.1 & 10 & 0.095 \\
\hline 298.16 & 796 & 160 & 0.1 & 20 & 0.079 \\
\hline 303.16 & 850 & 170 & 0.1 & 10 & 0.082 \\
\hline 308.16 & 925 & 175 & 0.2 & 10 & 0.093 \\
\hline 313.16 & 1000 & 180 & 0.2 & 10 & 0.081 \\
\hline
\end{tabular}

Table $3 \mathrm{~A}$ : The value of $\mathrm{Ln} \mathrm{K}$ and $1 \backslash \mathrm{T}$ for $[\mathrm{Ni}-4-\mathrm{AAp}] \mathrm{Cl}_{2}$ in different mixed solvent

\begin{tabular}{|c|c|c|c|c|}
\hline $1 / \mathrm{T}$ & $\operatorname{Ln} K_{A} 100 \%$ & $\operatorname{Ln} K_{A} 90 \%$ & $\operatorname{Ln} K_{A} 70 \%$ & $\operatorname{Ln} K_{A} 50 \%$ \\
\hline 0.00341111 & 6.549651 & 6.618739 & 6.818924 & 7.003065 \\
\hline 0.0033539 & 6.476972 & 6.745236 & 6.949856 & 7.149917 \\
\hline 0.00329859 & 6.327937 & 6.849066 & 7.047517 & 7.244228 \\
\hline 0.00324507 & 6.159095 & 7.003065 & 7.149917 & 7.31322 \\
\hline 0.00319326 & 5.991465 & 7.090077 & 7.189168 & 7.377759 \\
\hline
\end{tabular}


Table $3 \mathrm{~B}$ : The value of $\mathrm{Ln} \mathrm{K}$ and $1 \backslash \mathrm{T}$ for $[\mathrm{Co}-4-\mathrm{AAp}] \mathrm{Cl}_{2}$ in different mixed solvent

\begin{tabular}{|c|c|c|c|c|}
\hline $1 / T$ & $\operatorname{Ln} K_{A} 100 \%$ & $\operatorname{Ln} K_{A} 90 \%$ & $\operatorname{Ln} K_{A} 70 \%$ & $\operatorname{Ln} K_{A} 50 \%$ \\
\hline 0.00341111 & 6.476972 & 7.049255 & 6.802395 & 6.829794 \\
\hline 0.0033539 & 6.588926 & 7.089243 & 6.907755 & 6.907755 \\
\hline 0.00329859 & 6.672033 & 7.129298 & 7.003065 & 6.549651 \\
\hline 0.00324507 & 6.745236 & 7.17012 & 7.047517 & 6.679599 \\
\hline 0.00319326 & 6.802395 & 7.20786 & 7.090077 & 6.745236 \\
\hline
\end{tabular}

Table $4 \mathrm{~A}$ : Thermodynamic parameters from the ion association constant in different temperatures and mixed solvents for the complex $\left[\mathrm{Ni}(4-\mathrm{AAP})_{3}\right] \mathrm{Cl}_{2}$.

\begin{tabular}{|c|c|c|c|}
\hline $\mathbf{T} /\left(\mathbf{K}^{\mathbf{0}}\right)$ & $-\Delta \mathrm{H}\left(\mathrm{kJmol}^{-1}\right)$ & $-\Delta \mathrm{G}\left(\mathrm{kJmol}^{-1}\right)$ & $-\Delta S\left(J^{-1} \mathrm{~mol}^{-1}\right)$ \\
\hline \multicolumn{4}{|c|}{$100 \%$ Methanol } \\
\hline 293.16 & 0.314 & 15.965 & 53.387 \\
\hline 298.16 & 0.314 & 16.063 & 52.821 \\
\hline 303.16 & 0.314 & 15.955 & 51.593 \\
\hline 308.16 & 0.314 & 15.782 & 51.022 \\
\hline 313.16 & 0.314 & 15.296 & 48.799 \\
\hline \multicolumn{4}{|c|}{$90 \%$ Methanol } \\
\hline 293.16 & 0.261 & 16.135 & 55.928 \\
\hline 298.16 & 0.261 & 16.733 & 56.996 \\
\hline 303.16 & 0.261 & 17.265 & 57.811 \\
\hline 308.16 & 0.261 & 17.934 & 59.044 \\
\hline 313.16 & 0.261 & 18.460 & 59.781 \\
\hline \multicolumn{4}{|c|}{$70 \%$ Methanol } \\
\hline 293.16 & 0.214 & 16.623 & 57.433 \\
\hline 298.16 & 0.214 & 17.228 & 58.499 \\
\hline 303.16 & 0.214 & 17.769 & 59.319 \\
\hline 308.16 & 0.214 & 18.319 & 60.141 \\
\hline 313.16 & 0.214 & 18.772 & 60.627 \\
\hline \multicolumn{4}{|c|}{$50 \%$ Methanol } \\
\hline 293.16 & 0.201 & 17.061 & 58.883 \\
\hline 298.16 & 0.201 & 17.724 & 60.119 \\
\hline 303.16 & 0.201 & 18.248 & 60.856 \\
\hline 308.16 & 0.201 & 18.729 & 61.429 \\
\hline 313.16 & 0.201 & 19.215 & 62.000 \\
\hline
\end{tabular}


Table 4 B: Thermodynamic parameters from the ion association constant in different temperatures and mixed solvents for the complex $\left[\mathrm{Co}(4-\mathrm{AAP})_{3}\right] \mathrm{Cl}_{2}$.

\begin{tabular}{|c|c|c|c|}
\hline $\mathbf{T} /\left(\mathbf{K}^{\mathbf{0}}\right)$ & $-\Delta \mathrm{H}\left(\mathrm{kJmol}^{-1}\right)$ & $-\Delta \mathrm{G}\left(\mathrm{kJmol}^{-1}\right)$ & $-\Delta \mathrm{S}\left(\mathrm{JK}^{-1} \mathrm{~mol}^{-1}\right)$ \\
\hline \multicolumn{4}{|c|}{$100 \%$ Methanol } \\
\hline 293.16 & 12.114 & 15.794 & 95.197 \\
\hline 298.16 & 12.114 & 16.336 & 95.419 \\
\hline 303.16 & 12.114 & 16.812 & 95.414 \\
\hline 308.16 & 12.114 & 17.294 & 95.430 \\
\hline 313.16 & 12.114 & 17.705 & 95.219 \\
\hline \multicolumn{4}{|c|}{$90 \%$ Methanol } \\
\hline 293.16 & 6.041 & 17.195 & 79.260 \\
\hline 298.16 & 6.041 & 17.575 & 79.207 \\
\hline 303.16 & 6.041 & 17.971 & 79.206 \\
\hline 308.16 & 6.041 & 18.370 & 79.215 \\
\hline 313.16 & 6.041 & 18.772 & 79.234 \\
\hline \multicolumn{4}{|c|}{$70 \%$ Methanol } \\
\hline 293.16 & 0.158 & 16.574 & 57.075 \\
\hline 298.16 & 0.158 & 17.129 & 57.979 \\
\hline 303.16 & 0.158 & 17.643 & 58.718 \\
\hline 308.16 & 0.158 & 18.062 & 59.125 \\
\hline 313.16 & 0.158 & 18.460 & 59.452 \\
\hline \multicolumn{4}{|c|}{$50 \%$ Methanol } \\
\hline 293.16 & 0.191 & 15.965 & 55.109 \\
\hline 298.16 & 0.191 & 16.559 & 56.178 \\
\hline 303.16 & 0.191 & 17.013 & 56.749 \\
\hline 308.16 & 0.191 & 17.499 & 57.405 \\
\hline 313.16 & 0.191 & 17.991 & 58.059 \\
\hline
\end{tabular}

If Stokes law were obeyed in a system, the value of the Walden product $\left(\eta_{0} \Lambda_{0}\right)$ would be constant only if the effective radius of the ion remains constant in the different media. He suggested that the major deviation in the Walden product is due to the variation of the electrochemical equilibrium between ions and the solvent molecules depending on the composition of the mixed polar solvents (Hemes, 1974). This is the case in the behaviour of the present system as indicated in Figure (4) where the ions suffer various degrees of salvation with different mixtures of methanol and water as described by (Hameed et al., 2008). Table (5) shows some physical constants of methanol-water mixtures and the Walden product $\left(\eta \Lambda_{0}\right)$ for this electrolyt,the decrease of the Walden product values indicates strong solvent-solute interaction as a result of which the solvent fails to decrease the mobility of the ions and increase the viscosity of the medium (Akrawi, 2002). 
Table 5: Dielectric constants (D), viscosities $(\eta)$ of methanol-watermixtures at $298 \mathrm{~K}$ and the Walden product $\left(\eta \Lambda_{\mathrm{o}}\right)$.

\begin{tabular}{|c|c|c|c|}
\hline Memetheth \% & D & $\eta$ (cp) & $\begin{array}{c}\text { Walden product } \\
\left(\eta \Lambda_{\mathbf{o}}\right)\end{array}$ \\
\hline 100 & 32.64 & 0.545 & 0.654 \\
\hline 90 & 37.23 & 0.575 & 0.460 \\
\hline 70 & 46.42 & $0 . .650$ & 0.780 \\
\hline 50 & 55.40 & 0.720 & 0.360 \\
\hline
\end{tabular}

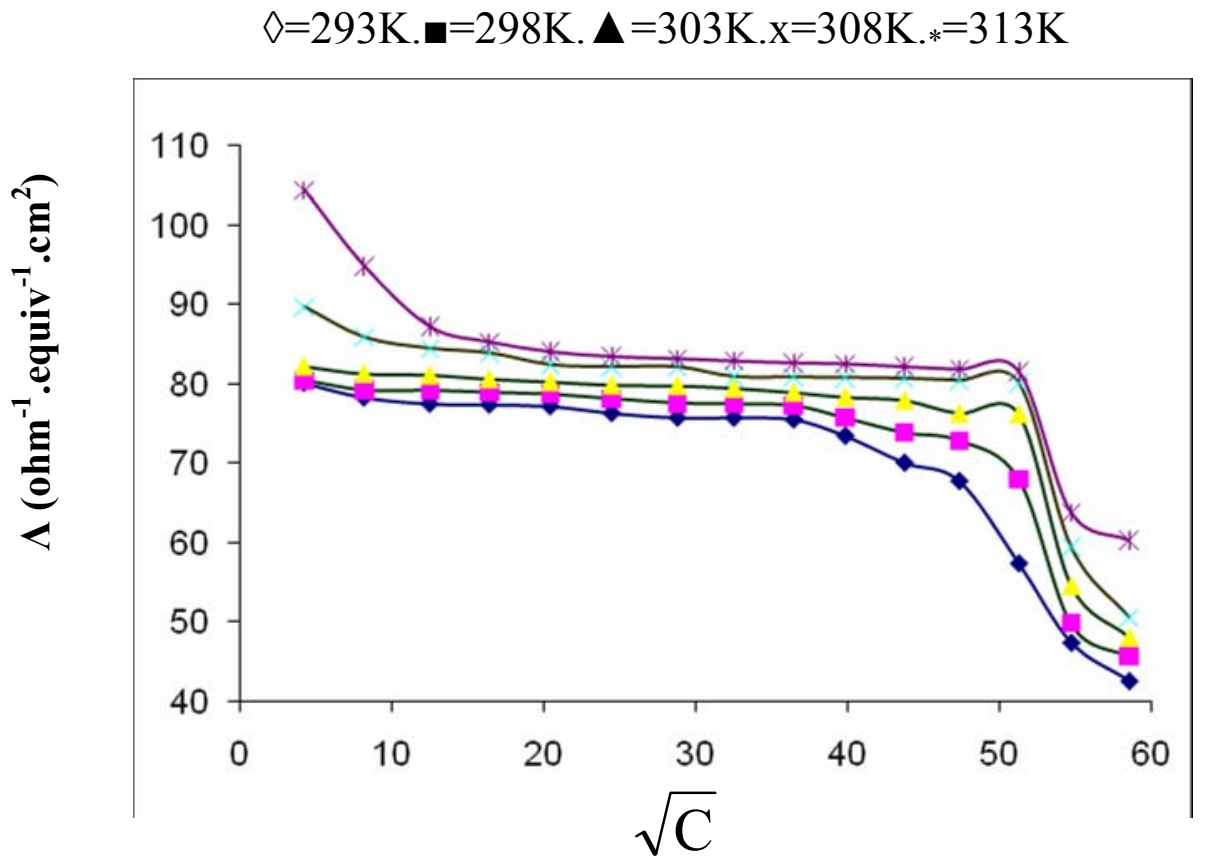

Fig. 1 A: Relation between equivalent conductance and square root of concentration of $[\mathrm{Ni}-4-\mathrm{AAp}] \mathrm{Cl}_{2}$ in $50 \%$ methanol -water mixture. 


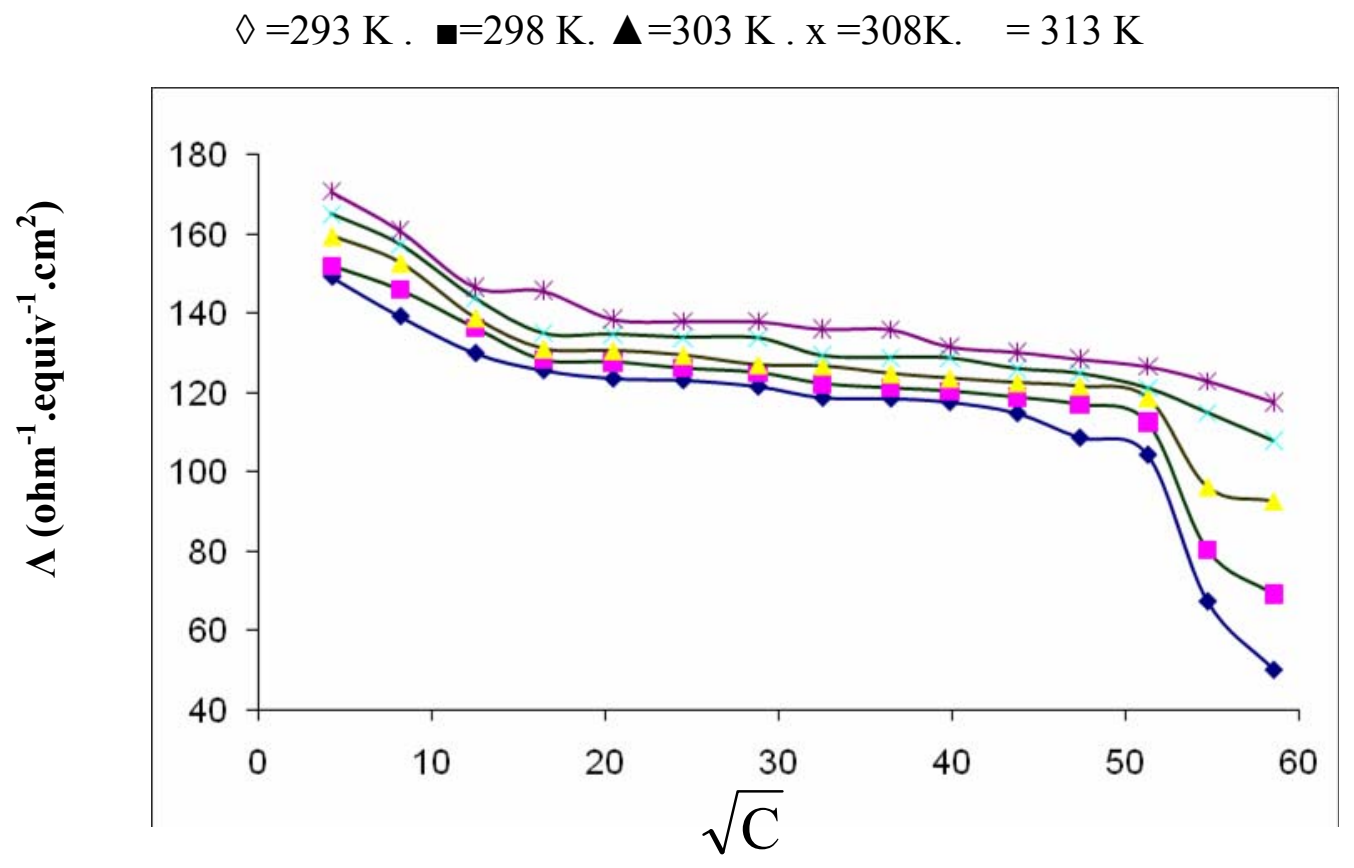

Fig. 1 B: Relation between equivalent conductance and square root of concentration of [Ni-4$\mathrm{AAp}] \mathrm{Cl}_{2}$ in $70 \%$ methanol -water mixture.

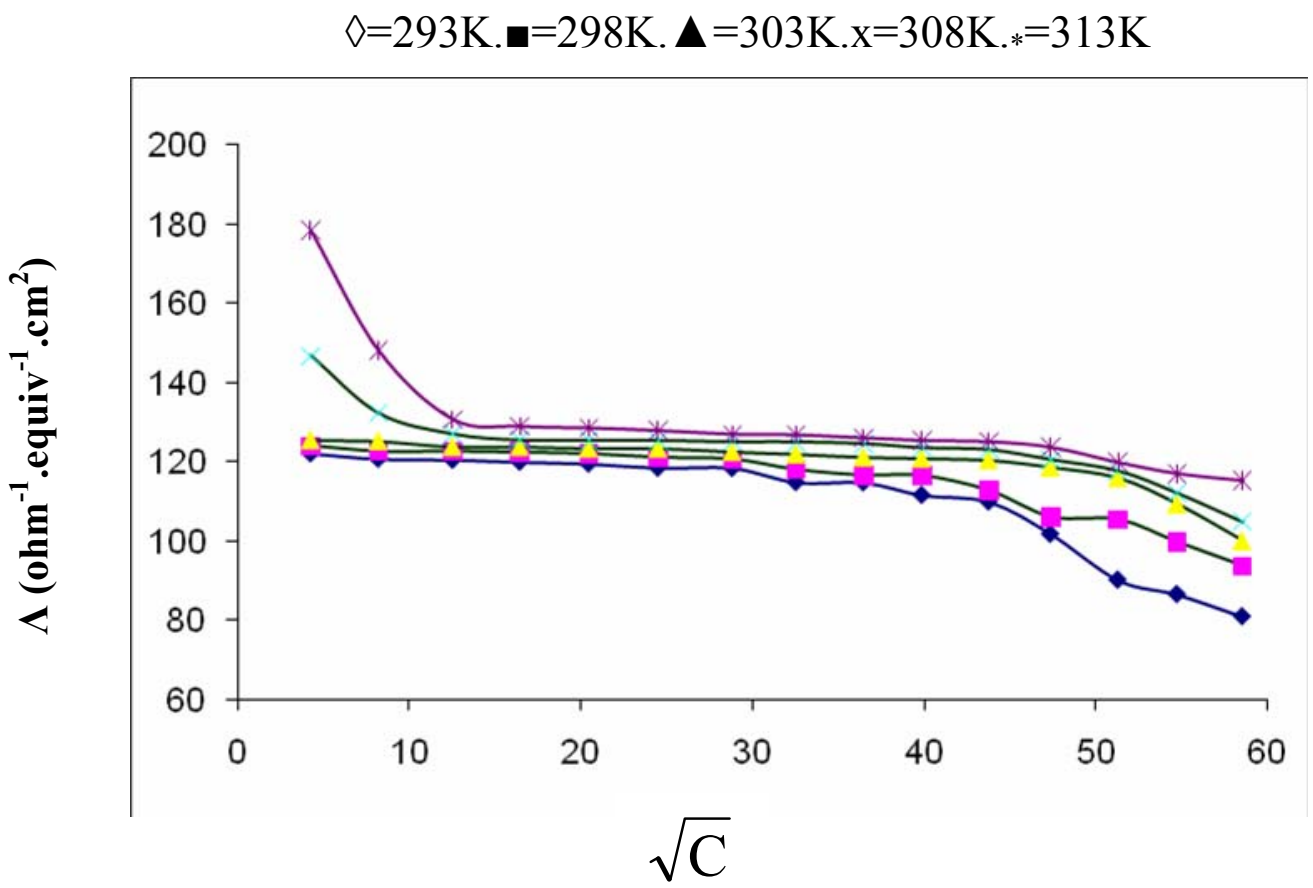

Fig. $1 \mathrm{C}$ : Relation between equivalent conductance and square root of concentration of $[\mathrm{Ni}-4-\mathrm{AAp}] \mathrm{Cl}_{2}$ in $90 \%$ methanol -water mixture. 
$\diamond=293 \mathrm{~K} . \boldsymbol{m}=298 \mathrm{~K} . \boldsymbol{\Delta}=303 \mathrm{~K} . \mathrm{x}=308 \mathrm{~K} . *=313 \mathrm{~K}$

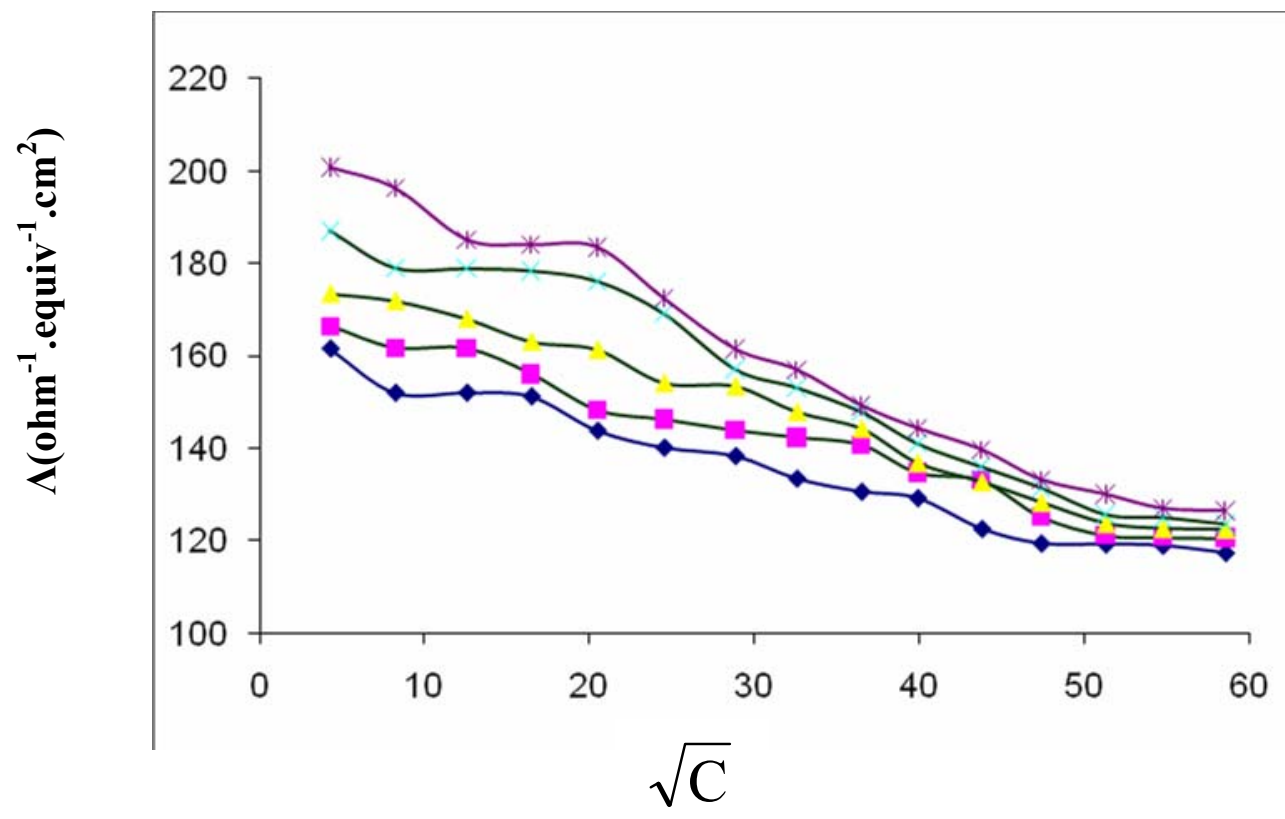

Fig. 1 D: Relation between equivalent conductance and square root of concentration of $[\mathrm{Ni}-4-\mathrm{AAp}] \mathrm{Cl}_{2}$ in $100 \%$ methanol.

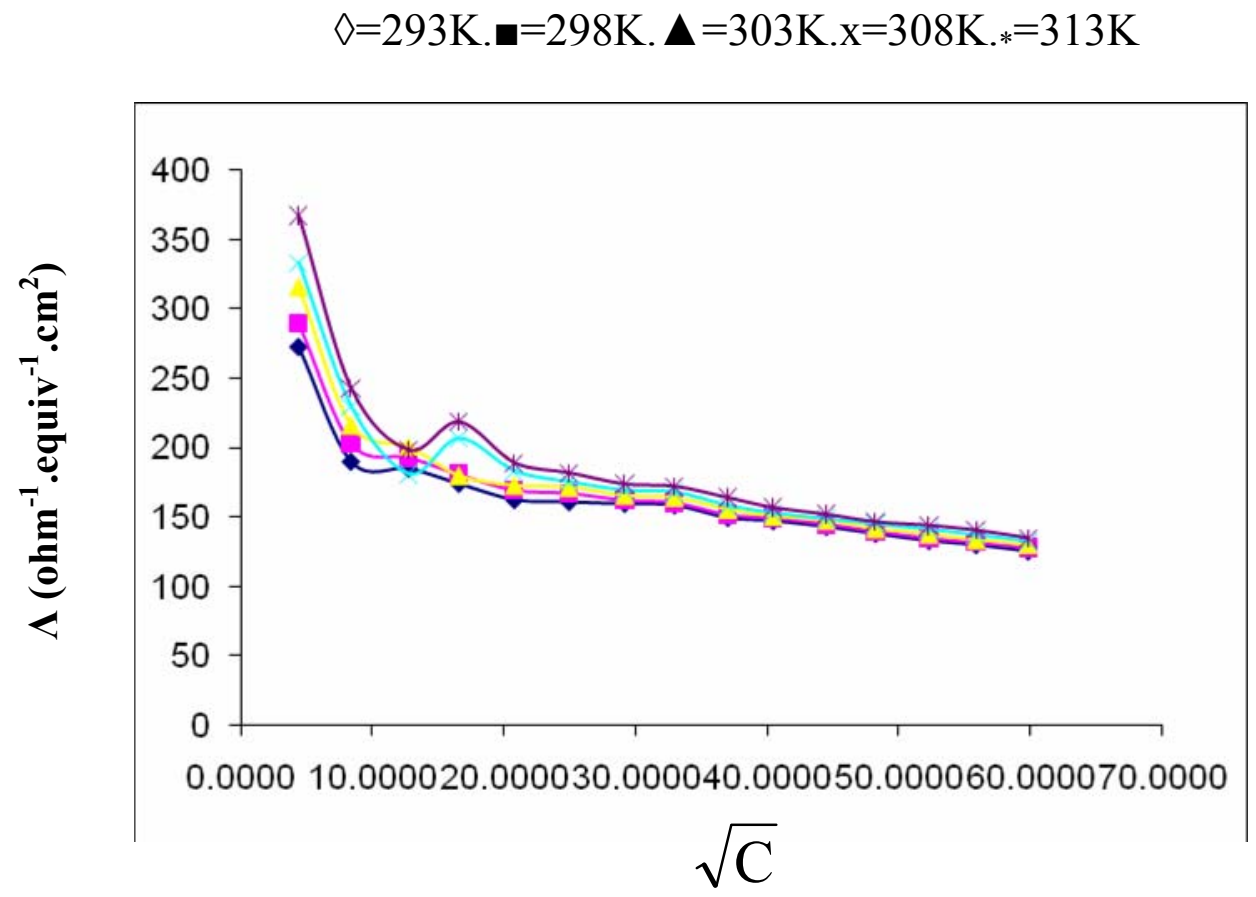

Fig. $1 \mathrm{E}$ : Relation between equivalent conductance and square root of concentration of $[\mathrm{Co}-4-\mathrm{AAp}] \mathrm{Cl}_{2}$ in $50 \%$ methanol -water mixture. 


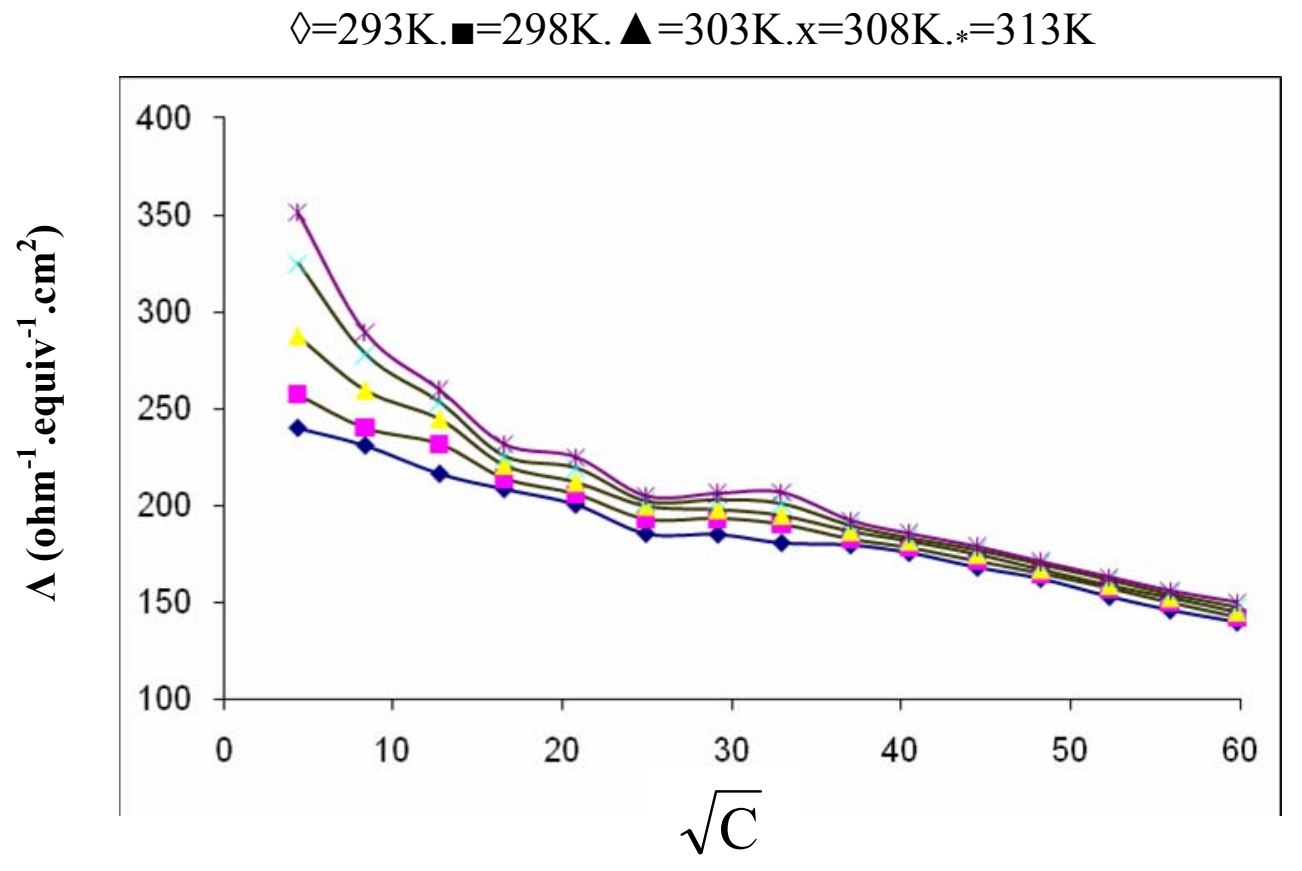

Fig. $1 \mathrm{~F}$ : Relation between equivalent conductance and square root of concentration of [Co-4-AAp $] \mathrm{Cl}_{2}$ in $70 \%$ methanol -water mixture

$$
\diamond=293 \mathrm{~K} . \boldsymbol{m}=298 \mathrm{~K} . \boldsymbol{\Delta}=303 \mathrm{~K} . \mathrm{x}=308 \mathrm{~K} . * 313 \mathrm{~K}
$$

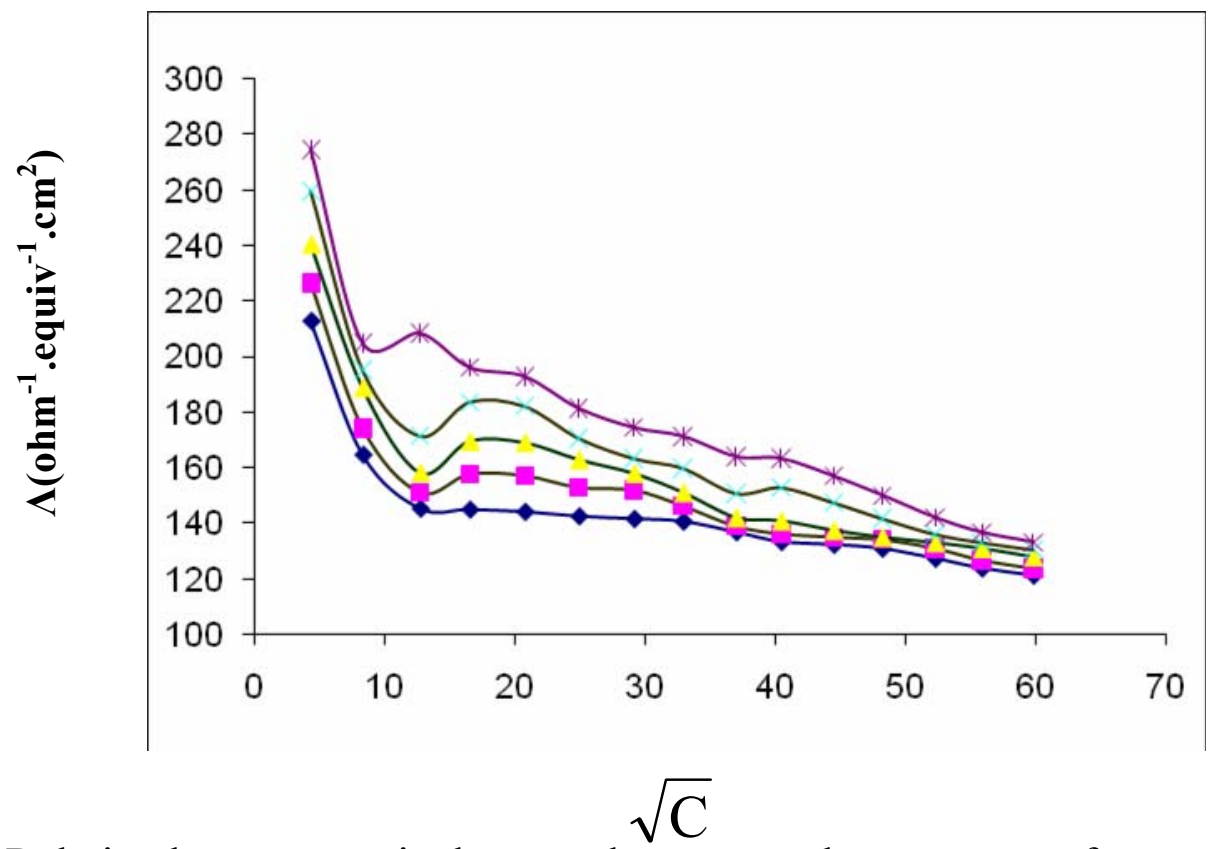

Fig. $1 \mathrm{G}$ : Relation between equivalent conductance and square root of concentration of [Co-4-AAp] $\mathrm{Cl}_{2}$ in $90 \%$ methanol -water mixture 


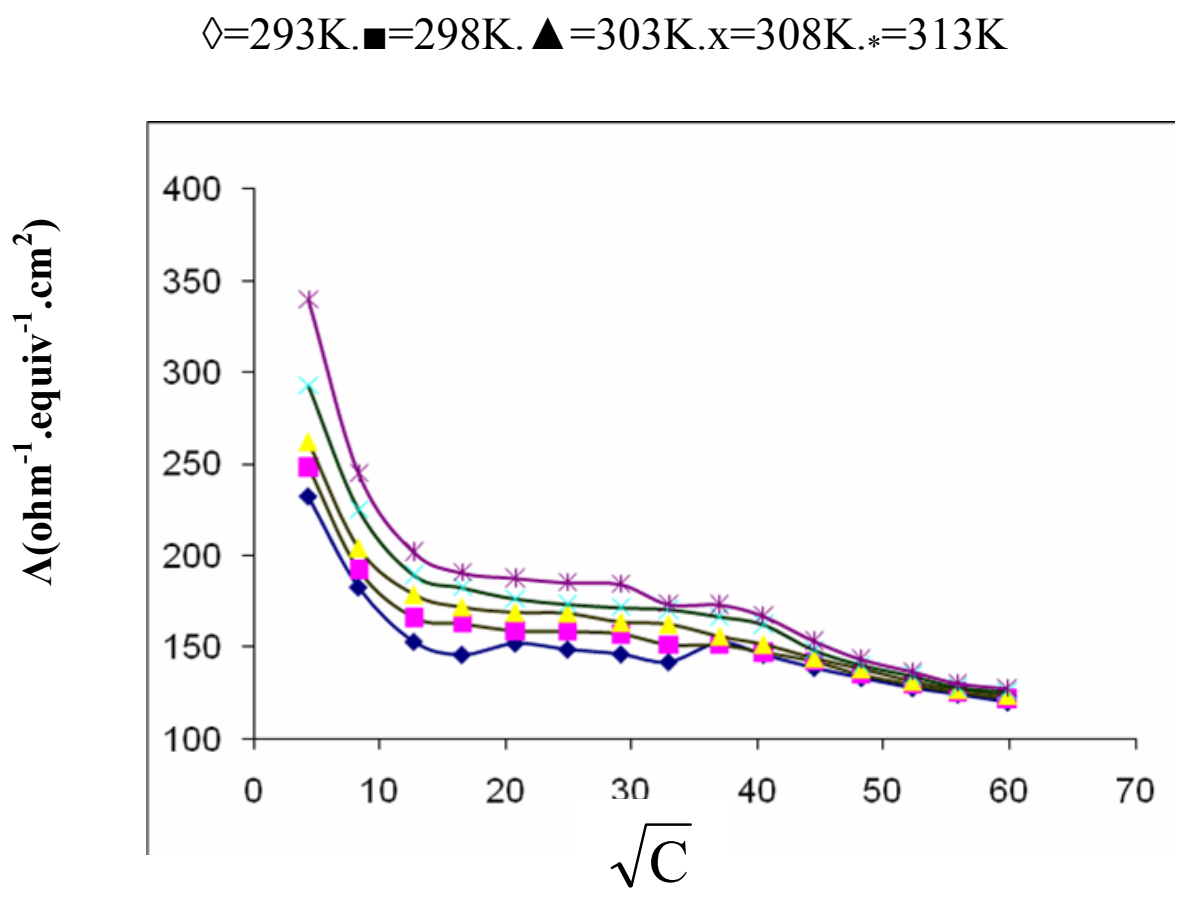

Fig. $1 \mathrm{H}$ : Relation between equivalent conductance and square root of concentration of [Co-4-AAp $] \mathrm{Cl}_{2}$ in $100 \%$ methanol.

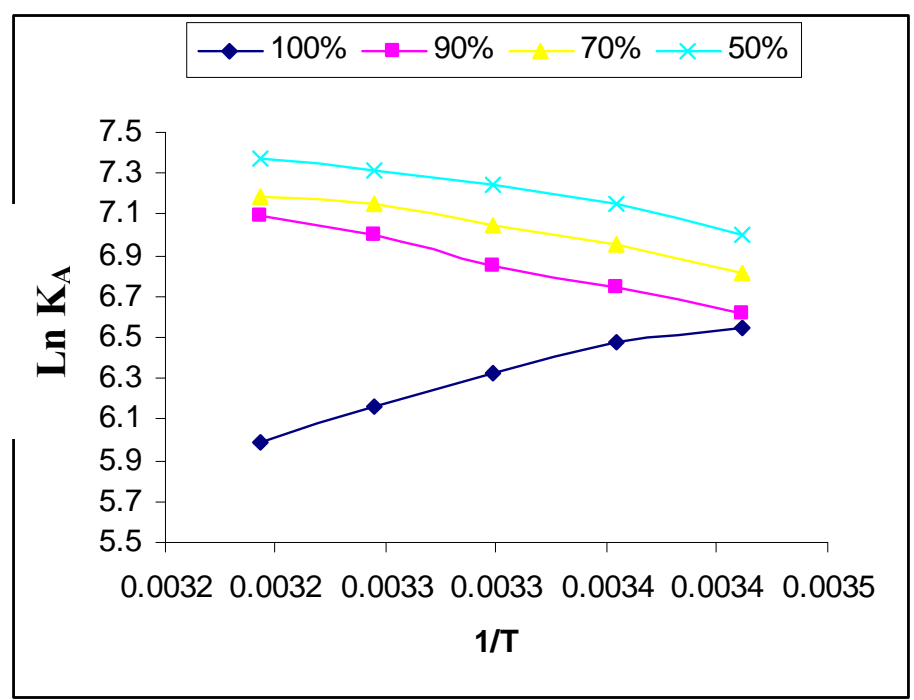

Fig. 2: Relation between $\mathrm{LnK}_{\mathrm{A}}$ and $1 \backslash \mathrm{T}$ for $[\mathrm{Ni}-4-\mathrm{AAp}] \mathrm{CL}_{2}$ in different percentages of methanol water. 


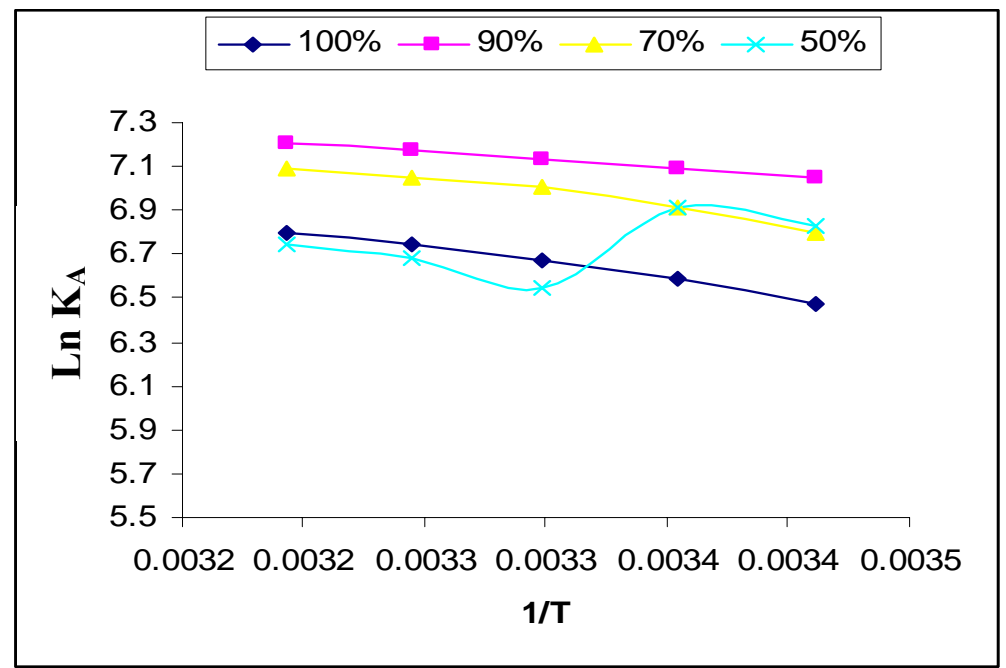

Fig. 3: Relation between $\mathrm{LnK}_{\mathrm{A}}$ and $1 \backslash \mathrm{T}$ for [Co-4-AAp]CL $\mathrm{L}_{2}$ in different percentages of methanol water.

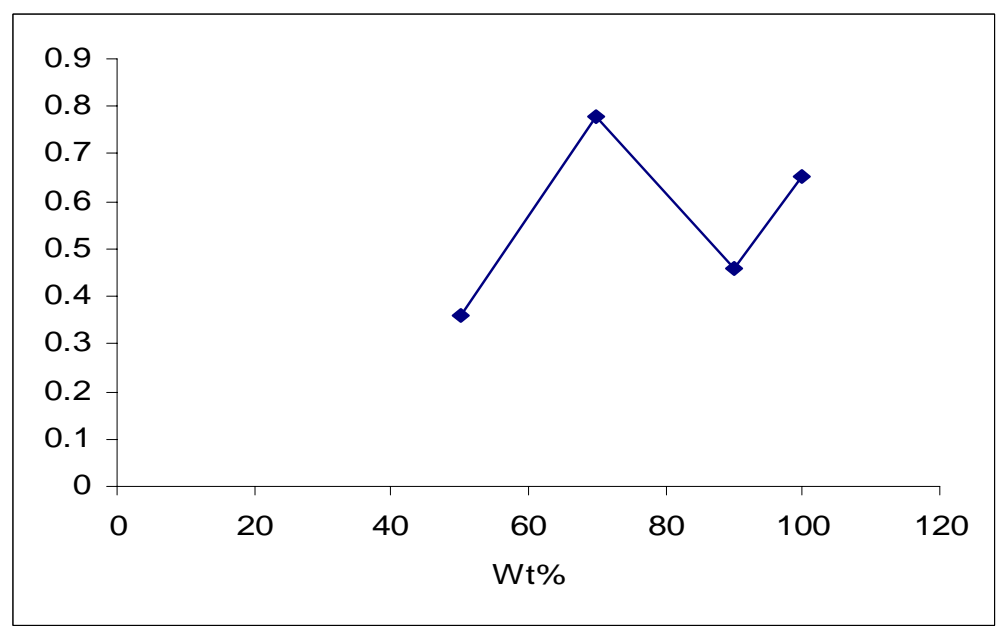

Fig. 4 : The plot of Walden against the weight percent of methanol water mixture

\section{REFERENCES}

Akrawi, B.A. (2002). The electrical conductivity of 2-(2,4-dichlorophenoxymethyl)-5-(3chlorophenyl)1,3,4-Oxadizle in methanol-water mixture at $25^{\circ} \mathrm{C}$. J. Raf. Sci. 13(1), 63-70.

Akrawi, B.; Hameed, Y. O. (2008). The electrical conductivity of $\left[\mathrm{Ni}(\mathrm{Phen})_{3}\right] \mathrm{Cl}_{2}$ in methanol-water mixtures at 298. 16K. National. J. Chem., 31, 447-454.

Dabbugh, A.M; Akrawi, B; Hameed, Y. O. (1996). Densities, viscosities and electrical conductivities of barium chloride in dimethyl sulfoxide and dimethyl formamide solutions. Mutah Lil-Buhooth WA Al-Dirasat. 11(5).

Daniels, F.; Williams, J. W.; Bender, P.; Alberty, R. A.; Cornwell, J. E.; Harriman, J. E.; (1970). " Experimental Physical Chemistry". 7th edn. McGraw=Hill, 51 p. 
Dawood, A. M. (1996). An application of Lee -Wheaton conductance equation to single and mixed symmetrical and unsymmetrical electrolytes., Ph.D. Thesis, University of Mosul.

Fukuda, Y.; Sone, K.; (1972). Studies on mixed chelates. I. Mixed copper (II) chelates with N.N.N`, N`-tetramethyl ethylenediamine and bidentate ligand. Bull. Chem. Soc. Jap. 45, 465-469.

Hamee, Y. O.; Thanon, F. A.; Abdul-Rahman, S. H. (2008). Electrical behavior of association phenomena of tetra aqua-1,10-phenanthroline Iron (II) Chloride in Methanol-Water Mixtures at different temperatures . J. Edu. and Sci., 21(3).

Hemes, P. (1974). The influence of true chemical equilibrium on the viscosity mobility product of electrolytes. J. Phys. Chem. 78, 907-909

Hikmat, N. A (2002). Conductance investigation of ion-pair formation by sodium acetate in methanol-water mixture. Iraqi J. Chem. 28(1), 97

Ito, K.; Iwamoto, I. ; Yamahato, Y. (1982). Effect of N-methylated diamine on ion pair formation of bis (2,4-pentanedionato)(diamine) cobalt (III) bromides in nitrobenzene by conductivity. Bull. Chem. Soc. Jpn. 55, 3190-3192.

Iwamoto, E.; Yamahato, Y. (1977). Stereospecific interaction between $\mathrm{Cr}(\mathrm{acac})_{3}$ or $\mathrm{Co}(\mathrm{acac})_{3}$ and $\left.\Delta-(-)_{589}-\mathrm{Ni}(\mathrm{Phen})_{3}\right)^{2+}$ in aqueous solution. Inorganic Nucl. Chem. Lett. 13, 399-402.

Jonan, I. S.; Ammar, A. A.; Amena, F.S. (2011). Synthesis and characterization of new complexes of anticipated analgesic activity formed by trasition metals $\mathrm{Co}(\mathrm{II}), \mathrm{Ni}$ (II) and $\mathrm{Cu}(\mathrm{II})$ with 4-amino antipyrine [4-AAP]. Accepted for publication in $\mathrm{Al}$ Taquani $J$.

Lee, W.H.; Wheaton R. J. (1978). Conductance of symmetrical, unsymmetrical and mixed electrolytes J. Chem. Soc., Faraday Trans, 11(74), 743-765.

Newmann, S.; Blinn, E.; Carison, L. (1979). 5,7,7,12,14,14-hexamethyl-1,4,8,11tetraazacyclotetradeca-4,11-diene) nickel (II) sulfate dehydrate in water-dioxane solution. J. Phys. Chem. 83, 676-680.

Perrin, D. D.; Armarego, W. L. F.; Perrin, D. R. (1966). "Purification of Laboratory Chemicals". 1st edn, Pergamon Press, Oxford, 199p.

Tsierkezos, N.; Molinou, I. (2007). Conductivity studies of n-Tetrabutylammonium Tetraphenylborate in 3-pentanone in the temperature range from 283.15 to 329.15K. J. Solution Chem., 36(2), 153-170.

Tominago, T. (1975). Solute-solvent interaction of metal chelate and onium electrolytes by study of viscosity and apparent molal volume in methanol, acetone and nitrobenzene. J. Phys. Chem. 79(16), 1664-1670.

Von Meter, M.; Newman, H. M. (1976). Solvation of the tris (1,10-phenanthroline) iron (II0 cation as measured by solubility and nuclear magnetic resonance shifts. $J$. Amer. Chem. Soc. 98, 1382-1388.

Yokoyoma, H.; Kon, H. (1988). Temperature dependence of hydrophobic ion association of Tris (1,10-phenanthroline) iron(II) ion with arene disulfonate ions in water . $J$. Phys. Chem. 95, 8956-8963. 